\section{Growth of melanoma brain tumors monitored by photoacoustic microscopy}

\author{
Jacob Staley, ${ }^{\text {a }}$ Patrick Grogan, ${ }^{\text {b }}$ Abbas K. Samadi, \\ Huizhong Cui, ${ }^{a}$ Mark S. Cohen, ${ }^{\text {b }}$ and Xinmai Yanga, \\ a University of Kansas, Department of Mechanical \\ Engineering, The KU Bioengineering Research Center, \\ Lawrence, Kansas 66045 \\ ${ }^{\mathrm{b}}$ University of Kansas Medical Center, Department of \\ Surgery, Kansas City, Kansas 66160
}

\begin{abstract}
Melanoma is a primary malignancy that is known to metastasize to the brain and often causes death. The ability to image the growth of brain melanoma in vivo can provide new insights into its evolution and response to therapies. In our study, we use a reflection mode photoacoustic microscopy (PAM) system to detect the growth of melanoma brain tumor in a small animal model. The melanoma tumor cells are implanted in the brain of a mouse at the beginning of the test. Then, PAM is used to scan the region of implantation in the mouse brain, and the growth of the melanoma is monitored until the death of the animal. It is demonstrated that PAM is capable of detecting and monitoring the brain melanoma growth noninvasively in vivo. $\odot 2010$ Society of Photo-Optical Instrumentation Engineers. [DOI: 10.1117/1.3478309]
\end{abstract}

Keywords: brain melanoma; photoacoustic microscopy.

Paper 10272LR received May 21, 2010; revised manuscript received Jul. 14, 2010; accepted for publication Jul. 15, 2010; published online Aug. 13, 2010

\section{Introduction}

Malignant melanoma is one of the most lethal cancers and represents a significant public health problem in the United States. For patients with stage- 4 melanoma, the incidence of brain metastases has been reported to be 10 to $40 \%$, making melanoma the third most common metastatic brain tumor in the United States, following lung cancer and breast cancer. ${ }^{1}$ With effective treatments, survival time following central nervous system (CNS) metastases is between 2 and 5 months. ${ }^{2-5}$ Without treatments, most patients show enhanced deterioration and ultimately death. Recognition of the initiation and progression of melanoma brain metastases in experimental models is necessary to identify new research strategies useful for the diagnosis and subsequent therapy of these tumors in humans. Hence, in vivo monitoring of the formation and growth of melanoma tumors in live brain could have major implications for the design of improved therapies.

In this study, we utilize the photoacoustic microscopy (PAM) technique to detect the progress of melanoma brain tumors in mice models in vivo. In photoacoustic (also called optoacoustic or thermoacoustic) imaging, short pulses of laser light are transmitted into the targeted area. The produced ultrasonic waves by photoacoustic effect are then detected by

Address all correspondence to: Xinmai Yang; E-mail: xmyang@ku.edu.

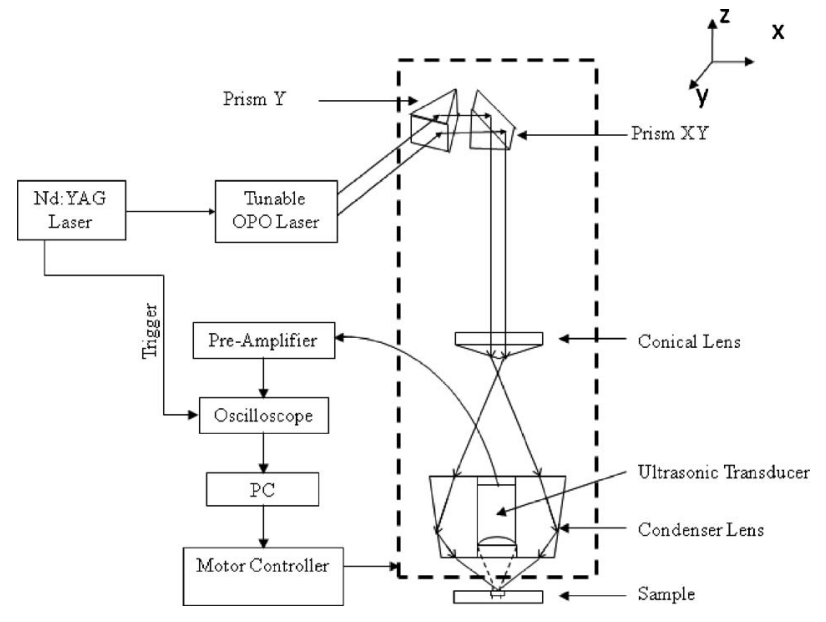

Fig. 1 Schematic of the PAM system.

ultrasonic transducers and reconstructed to form images representing optical absorption. Since melanin has high optical absorption (e.g., 1000 times that of water at 700-nm wavelength), significant contrast between melanoma and background tissue can be achieved using photoacoustic imaging. Compared with imaging modalities such as computed tomography $(\mathrm{CT})$, magnetic resonance imaging (MRI), and positron emission tomography (PET), all of which have been used to detect melanoma brain metastases noninvasively in vivo, ${ }^{6,7}$ photoacoustic imaging is low cost, using nonionizing radiation and based on sharp optical contrast. In contrast to highresolution, purely optical imaging methods, ${ }^{8}$ photoacoustic imaging can image much deeper and has no requirements for a cranial window.

Photoacoustic imaging has been applied to detect skin melanomas in small animal models. ${ }^{9,10}$ Real-time detection of circulating melanoma cells using photoacoustic flow cytometry has also been proposed. ${ }^{11-15}$ In this work, we demonstrate the capability of photoacoustic imaging for noninvasive monitoring of the growth of melanoma brain tumors in a mouse model.

\section{Methods and Materials}

Our photoacoustic imaging system is identical to the one presented by Song et al. for deep photoacoustic imaging. ${ }^{16}$ The system (Fig. 1) consists of an ultrasound transducer, a laser system, and receiving electronics. A Q-switched Nd:YAG laser (Surelite, Continuum, Santa Clara, California) was used to pump a tunable OPO laser (Surelite OPO PLUS, Continuum, Santa Clara, California) to obtain a 764-nm wavelength laser with a $10-\mathrm{Hz}$ pulse repetition rate. The produced laser light forms a ring-shaped illumination after passing through two prisms and a conical lens, and then is refocused inside the tissue sample by an optical condenser. At the tissue surface, the ring has a diameter of $\sim 5 \mathrm{~mm}$ with a focal depth of $\sim 3 \mathrm{~mm}$ for current application. The subsequently generated ultrasonic waves are detected by a focused ultrasonic transducer (13-2506-R, Olympus-NDT, Waltham, Massachusetts), delivered to a preamplifier (5072PR, Olympus-NDT, Waltham, Massachusetts) with 30-dB gain, and finally col- 


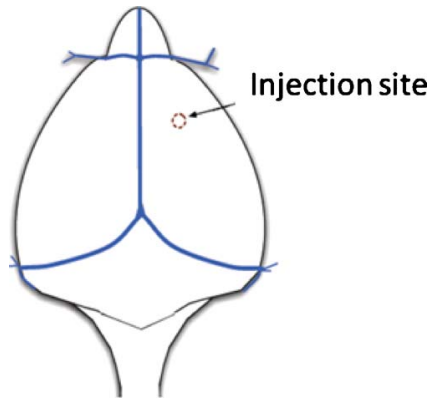

Fig. 2 Site of injection of B16F10 tumor cells in the in vivo mouse brain.

lected by a personal computer through an A/D Scope Card (CS21G8-256MS, Gage, Lockport, Illinois) with a 500-MHz sampling rate. The conical and condenser lenses are driven by a 3-D translation stage to enable the transducer to mechanically scan the targeted region. Two prisms are used to enable the optical beam folding for a 2-D mechanical scanning. Prism $\mathrm{Y}$ moves in the $y$ direction, whereas prism XY can move in both $x$ and $y$ directions. The ultrasound transducer (15-mm focal length, 9.4-mm aperture size) has a central frequency of $25 \mathrm{MHz}$ with $61 \%-6-\mathrm{dB}$ fractional bandwidth, and exhibits lateral resolution of $150 \mu \mathrm{m}$ and axial resolution of $100 \mu \mathrm{m}$ when used for photoacoustic imaging.

All in vivo imaging evaluations were done using an orthotopic tumor model for metastastic melanoma to the brain on a stereotactic protocol approved by the University of Kansas Institutional Animal Care and Use Committee. B16F10 melanoma tumor cells grown in Dulbecco's Modified Eagle Medium (DMEM) supplemented by $10 \%$ fetal bovine serum, $1 \%$ penicillin, and $1 \%$ streptomycin were harvested by trypsinization, washed in $1 \times$ phosphate-buffered saline, resuspended in $1 \times$ phosphate-buffered saline at a concentration of 2500 cells $/ \mu \mathrm{L}$, and kept on ice until injected intracranially. $\mathrm{Balb} / \mathrm{C}$ mice were deeply anesthetized using a mixture of 87-mg/kg ketamine and 13-mg/kg xylazine via an intraperitoneal injection. The hairs on the top of the animals' heads were shaved to expose the skin, and animals were then appropriately positioned in a small animal stereotactic frame. Following liquid tear application to the eyes to prevent ocular damage during anesthesia, povidone iodine was applied on the skin covering the skull around the anticipated midline incision site, and an alcohol pad was then used to wipe down the skin to remove excess povidone iodine and provide further sterilization. The calvarium was exposed via a midline incision of the scalp $\sim 1 \mathrm{~cm}$ in length, and a burr hole was drilled $1 \mathrm{~mm}$ anterior and $2 \mathrm{~mm}$ lateral (right) from the bregma by a Dremel 10.8V 8000-03 with a rounded high-speed cutter bit. Figure 2 shows the cell injection site. A $10-\mu \mathrm{L}$ blunt-tipped Hamilton syringe loaded with $2 \mu \mathrm{L}$ was lowered into the hole, $3.3 \mathrm{~mm}$ from the cortical surface but retracted $0.3 \mathrm{~mm}$ to form a small pocket for the liquid to be injected, thereby introducing the cells into the right basal ganglia. The B16F10 cells were injected into the brain at a speed of $0.4 \mu \mathrm{L} / \mathrm{min}$ and a total volume of $2 \mu \mathrm{L}$ (5000 cells injected per mouse). The syringe was left in place for $\sim 1$ min following tumor cell injection to allow for pressure stabilization. After the intracranial injection, each animal was removed from the stereotac-
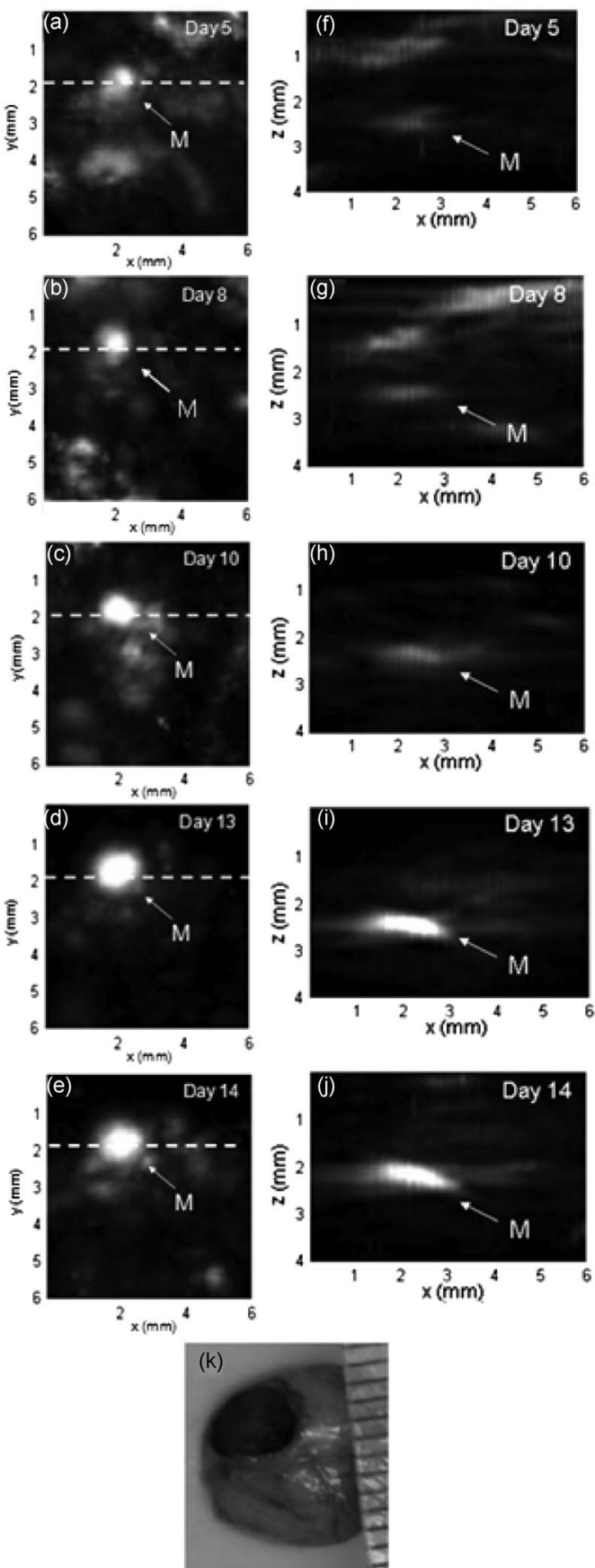

Fig. 3 Noninvasive MAP images of the brain melanoma in (a) day 5, (b) day $8,(\mathrm{c})$ day $10,(\mathrm{~d})$ day 13 , and (e) day 14 , after the injection of tumor cells, respectively. (f) through (j) show the B-scan images corresponding to the dashed lines in (a) through (e), respectively. $(\mathrm{k})$ Invasive anatomical photograph after the mouse death. All photoacoustic images are in the same intensity scale. $M$ is the melanoma brain tumor.

tic frame, and the incision was closed with a surgical suture. Triple antibiotic ointment was added to the incision to help prevent infection, and the animals were slowly warmed on a heating pad and monitored until they awakened.

Photoacoustic images of the brain melanoma were first acquired on the fifth day after the tumor cell injection. During photoacoustic imaging, a mouse with a melanoma brain tumor 
was initially anesthetized with a mixture of $87-\mathrm{mg} / \mathrm{kg}$ ketamine and $13-\mathrm{mg} / \mathrm{kg}$ xylazine, and then the anesthesia was maintained with the inhalation of a mixture of pure oxygen and $1 \%$ isoflurane. The brain region where the melanoma cells were injected was imaged. It took about 40 min scanning time to get one photoacoustic image. The same process was repeated the following days, and the growth of the brain melanoma tumor could be directly indicated from the photoacoustic images as the size of the tumor increased. The animals showed classic signs of morbidity approximately two weeks following the tumor injections.

\section{Results and Discussions}

Maximum amplitude projection (MAP) of the photoacoustic images of the melanoma in mouse brain, which are formed by projecting the maximum photoacoustic amplitudes along the $z$ axis to its orthogonal plane, are presented in Figs. 3(a)-3(e). Photoacoustic signals from the scalp and skull were removed in the MAP images to clearly show the tumor inside the brain. Figures 3(a)-3(e) were taken from an experimental animal on days $5,8,10,13$, and 14 after the injection of tumor cells, respectively. On days 5 and 8 , the brain tumor signals are relatively weak because the tumor is still small. From the photoacoustic image, the signal-to-noise ratio (SNR) of the tumor region is $15 \mathrm{~dB}$ at day 5 , and the tumor size is around $1 \mathrm{~mm}$ in diameter and $0.3 \mathrm{~mm}$ in depth. On day 10 , the photoacoustic signals from the melanoma brain tumor become dominate. The SNR of the tumor region increases to $26 \mathrm{~dB}$, and the size of the tumor increases to $2 \mathrm{~mm}$ in diameter and $0.7 \mathrm{~mm}$ in depth. The tumor continues to grow over the last two days before morbidity, as the SNR of the tumor region increases to $30 \mathrm{~dB}$, and the size is about $4 \mathrm{~mm}$ in diameter and over $1 \mathrm{~mm}$ in depth on day 14 .

The corresponding B-mode (cross sectional) photoacoustic images of the melanoma tumor from the same mouse are in Figs. 3(f)-3(j). These clearly show the depth of the melanoma tumor in the mouse brain on the five separate imaging days after injection. The brain melanoma was initially located about 2 to $3 \mathrm{~mm}$ deep underneath the skull. As the time elapses, the thickness of the melanoma brain increases. On day 14 , the mouse died from the brain melanoma, and an open skull photograph was taken, as shown in Fig. 3(k). The final size of the tumor is about $4 \mathrm{~mm}$ in diameter inside the brain.

Both size and depth information of brain tumors are important in planning and verifying the treatment of primary brain tumors (e.g., glioma). This study showed that PAM can monitor the brain tumor growth inside a small animal's brain noninvasively, and therefore photoacoustic imaging is capable of being an imaging tool to track in vivo the response of the brain tumor to therapies.

\section{Conclusions}

We use a reflection-mode PAM system with a $25-\mathrm{MHz}$ focused ultrasonic transducer, and demonstrate the capability of successfully detecting the progress of brain melanoma tumors in a metastasis model. This technique can be used to evaluate the brain melanoma tumor and its response to therapy in a small animal mode noninvasively.

\section{References}

1. N. Xu, H. Shu, R. Marcus, and I. R. Crocker, "Radiation necrosis after stereotactic radiosurgery for metastatic brain disease," Int. J. Radiat. Oncol., Biol., Phys. 69, S163-S163 (2007).

2. A. A. Tarhini and S. S. Agarwala, "Management of brain metastases, in patients with melanoma," Curr. Opin. Oncol. 16, 161-166 (2004).

3. Y. Mori, D. Kondziolka, J. C. Flickinger, J. M. Kirkwood, S. Agarwala, and L. D. Lundsford, "Stereotactic radiosurgery for cerebral metastatic melanoma: factors affecting local disease control and survival," Int. J. Radiat. Oncol., Biol., Phys. 42, 581-589 (1998).

4. R. A. Patchell, P. A. Tibbs, J. W. Walsh, R. J. Dempsey, Y. Maruyama, R. J. Kryscio, W. R. Markesbery, J. S. Macdonald, and B. Young, "A randomized trial of surgery in the treatment of single metastases to the brain," N. Engl. J. Med. 322, 494-500 (1990).

5. C. Yu, J. Chen, M. Apuzzo, S. O’Day, S. L. Giannotta, J. S. Weber, and Z. Petrovich, "Metastatic melanoma to the brain: prognostic factors after gamma knife radiosurgery," Int. J. Radiat. Oncol., Biol., Phys. 52, 1277-1287 (2002)

6. A. Panigrahy and S. Bluml, "Neuroimaging of pediatric brain tumors: from basic to Advanced magnetic resonance imaging (MRI)," $J$. Child Neurol. 24, 1343-1365 (2009).

7. Y. Mishima, Y. Imahori, C. Honda, J. Hiratsuka, S. Ueda, and T. Ido, "In vivo diagnosis of human malignant melanoma with positron emission tomography using specific melanoma-seeking F-18-DOPA analogue," J. Neuro-Oncol. 33, 163-169 (1997).

8. Y. Kienast, L. von Baumgarten, M. Fuhrmann, W. Klinkert, R. Goldbrunner, J. Herms, and F. Winkler, "Real-time imaging reveals the single steps of brain metastasis formation," Nat. Med. 16, 116U157 (2010).

9. J. T. Oh, M. L. Li, H. F. Zhang, K. Maslov, G. Stoica, and L. V. Wang, "Three-dimensional imaging of skin melanoma in vivo by dual-wavelength photoacoustic microscopy," J. Biomed. Opt. 11, 034032 (2006).

10. H. F. Zhang, K. Maslov, G. Stoica, and L. H. V. Wang, "Functional photoacoustic microscopy for high-resolution and noninvasive in vivo imaging," Nat. Biotechnol. 24, 848-851 (2006).

11. E. I. Galanzha, E. V. Shashkov, M. S. Kokoska, J. A. Myhill, and V. P. Zharov, "In vivo non-invasive detection of metastic melanoma in vasculature and sentinel lymph nodes by photoacoustic cytometry," Lasers Surg. Med. Suppl. 20, 81 (2008).

12. V. P. Zharov, E. I. Galanzha, E. V. Shashkov, N. G. Khlebtsov, and V. V. Tuchin, "In vivo photoacoustic flow cytometry for monitoring of circulating single cancer cells and contrast agents," Opt. Lett. 31, 3623-3625 (2006).

13. V. P. Zharov, E. I. Galanzha, E. V. Shashkov, J. Kim, N. G. Khlebtsov, and V. V. Tuchin, "Photoacoustic flow cytometry: principle and application for real-time detection of circulating single nanoparticles, pathogens, and contrast dyes in vivo," J. Biomed. Opt. 12, 051503 (2007).

14. E. I. Galanzha, E. V. Shashkov, V. V. Tuchin, and V. P. Zharov, "In vivo multispectral, multiparameter, photoacoustic lymph flow cytometry with natural cell focusing, label-free detection and multicolor nanoparticle probes," Cytometry, Part A 73A, 884-894 (2008).

15. W. L. Olszewski and A. Tarnok, "Photoacoustic listening of cells in lymphatics: research art or novel clinical noninvasive lymph test," Cytometry, Part A 73A, 1111-1113 (2008).

16. K. H. Song and L. V. Wang, "Deep reflection-mode photoacoustic imaging of biological tissue," J. Biomed. Opt. 12, 060503 (2007). 